DOI: https://doi.org/10.46296/gt.v5i9edespmar.0057

\title{
IDENTIFICACIÓN DE TRASTORNOS MENTALES PRESENTES EN MUJERES EMBARAZADAS EN LA CIUDAD DE ROCAFUERTE
}

\section{IDENTIFICATION OF MENTAL DISORDERS PRESENT IN PREGNANT WOMEN IN THE CITY OF ROCAFUERTE}

\author{
Gómez-Reyna Robert Rafael 1; Valdivieso-López Isabel Patricia ${ }^{2}$ \\ ${ }^{1}$ Estudiante de la Maestría Académica con Trayectoria en Investigación en Psicología, Mención \\ Psicoterapia, Instituto de Posgrado, Universidad Técnica de Manabí. Portoviejo, Ecuador. \\ Correo: robert.gomez@utm.edu.ec. ORCID ID: https://orcid.org/0000-0002-7898-0657 \\ 2 Docente investigadora de la Escuela de Psicología, Universidad Técnica de Manabí. \\ Portoviejo, Ecuador. Correo: isabel.valdiviezo@utm.edu.ec. \\ ORCID ID: https://orcid.org/0000-0003-4259-2019
}

\begin{abstract}
Resumen
Existen diversas investigaciones en las que demuestran múltiples factores de riesgo en las diferentes etapas del embarazo, los cuales influyen en el curso y aparición de los trastornos mentales. El objetivo es determinar la relación de los trastornos mentales en las mujeres embarazadas. Por lo cual se realiza un estudio desde el enfoque cuantitativo descriptivo, con tipo de diseño transversal. El universo corresponde a todas las mujeres embarazadas atendidas por control en el Hospital Básico Natalia Huerta de Niemes del Cantón Rocafuerte- Manabí. La muestra se obtuvo de la población de mujeres embarazadas atendidas en el hospital durante un trimestre. Se realizó la evaluación mediante la información de las fichas de las pacientes ingresadas a más de la aplicación del Cuestionario de Síntomas "Self-Reporting Questionnaire" (SRQ). Para el procesamiento de datos, se utilizó el software estadístico SPSS. La aplicación de psicoterapia es trascendental en el tratamiento a múltiples problemas de salud mental y su identificación e intervención temprana puede contribuir a un mejor manejo de estos cuadros clínicos. Se evidenció una prevalencia de antecedentes psico-sociales en las purpurea, con un alto índice de alteraciones de salud mental tales como ansiedad y depresión.
\end{abstract}

Palabras clave: factores de riesgo, embarazo, alteraciones mentales, gestantes, salud mental.

\begin{abstract}
There are various investigations that show multiple risk factors at different stages of pregnancy, which influence the course and appearance of mental disorders. The objective is to determine the relationship of mental disorders in pregnant women. Therefore, a study is carried out from a descriptive quantitative approach, with a type of cross-sectional design. The universe corresponds to all pregnant women treated by control at the Natalia Huerta de Niemes Basic Hospital in the Rocafuerte canton. The sample was obtained from the population of pregnant women treated in the hospital during one trimester. Clinical observation, the semi-structured interview and the application of the Symptom Questionnaire "Self-Reporting Questionnaire" (SRQ) were used. For data processing, the statistical software SPSS was used. The application of psychotherapy is transcendental in the treatment of multiple problems of mental health and its identification and early intervention can contribute to a better management of these clinical pictures. A prevalence of psychosocial antecedents was evidenced in the purpurea, with a high rate of mental health disorders such as anxiety and depression.
\end{abstract}

Keywords: pregnancy, mental disorders, pregnant, mental health.

Información del manuscrito:

Fecha de recepción: 06 de enero de 2022.

Fecha de aceptación: 24 de febrero de 2022.

Fecha de publicación: 04 de marzo de 2022. 


\section{INTRODUCCIÓN}

Existe una amplia variedad de trastornos mentales que pueden estar presentes en la vida de una persona, estos con distintos tipos de etiología y sintomatología. según la , estos síntomas se caracterizan por una combinación de alteraciones del pensamiento, la percepción, las emociones, la conducta y las relaciones con los demás.

Las diferentes etapas de la vida de las personas presentan distintos desafíos, y la etapa del embarazo no es la excepción, las mujeres se exponen a muchos cambios tanto fisiológicos como psicológicos, mismos que están asociados a una mayor fragilidad de su salud mental. En este periodo existen cambios en su organismo que alteran su psiquismo y su papel sociofamiliar (Guimaraes et al., 2019).

Durante el embarazo y el posparto, es común que aparezcan dificultades y trastornos psiquiátricos. Alrededor del $50 \%$ de los episodios psiquiátricos de este período son episodios debutantes, por lo que resulta necesario la búsqueda sistemática de los factores de vulnerabilidad en todas las mujeres embarazadas. Si previamente al embarazo existe un trastorno psiquiátrico, el riesgo de recaída varía entre el $30 \%$ y el $70 \%$ dependiendo de los trastornos y su evolución (Sutter-Dallay et al., 2020).

Existen diversos factores que se han asociado a la morbilidad psiquiátrica durante el embarazo. De manera general se pueden mencionar la gestación en la adolescencia, el embarazo no planificado, el estado civil, conflictos en la relación de pareja, ser multípara, un bajo nivel educativo y la carencia de apoyo social (Pérez-Solís et al., 2020). Sin dejar de lado otros aspectos como el estilo de vida, factores hereditarios y neuropsicológicos que pueden actuar como factores tanto predisponentes y precipitantes en la aparición de trastornos mentales como la depresión y ansiedad.

La Organización Panamericana de la Salud (2017), considera que la depresión ocupará el primero lugar dentro de la lista de trastornos que puedan afectar a la población. Se estima que hoy en día existen 340 millones de personas viviendo con un trastorno depresivo. Las mujeres son más propensas a presentar depresión en una proporción de 2:1 con relación a los hombres, siendo 
esta una de las principales causas de enfermedad en mujeres de 15 a 44 años a nivel mundial.

Otro de los trastornos que puede complicar a las mujeres en periodo de gestación es la ansiedad, esta puede llevar a las pacientes a presentar un cuadro de depresión (Sainz Aceves et al., 2013). La ansiedad es el segundo trastorno con más prevalencia a nivel mundial (Organización Panamericana de la Salud/OMS, 2020). Los episodios de ansiedad pueden interferir significativamente a nivel psicológico, familiar y social, y tienen repercusiones negativas en el embarazo, tanto para la madre como para el feto, además, puede interferir negativamente en la relación con su bebé y en el vínculo madre hijo. A pesar del impacto e importancia de los trastornos de ansiedad éstos suelen pasar desapercibidos, aun por el personal de salud de atención primaria. Estas alteraciones tienen una alta comorbilidad entre sí y con los estados depresivos (González, 2015).

La salud física de la madre también juega un papel fundamental, ya que durante el embarazo se pueden acentuar algunas condiciones de salud, por ejemplo, un tercio de las mujeres que padecen de epilepsia pueden experimentar un incremento de la frecuencia de sus crisis y las consecuencias de éstas están ligadas a múltiples afectaciones físicas que pueden interferir en el desarrollo de la madre y el hijo. A nivel psicológico, estas pueden llevar a incrementar los niveles de estrés y ansiedad de la mujer gestante (Nariño, 2017).

La psicoterapia, misma que se puede aplicar desde distintas corrientes psicológicas y que se divide en terapias de primera, segunda y tercera generación es una opción para el tratamiento de trastornos mentales. La mayoría de ellas se centran en objetivos específicos para la rehabilitación y tratamiento del individuo. Estas son una opción para las mujeres embarazadas, y que, si bien en nuestro país no existen protocolos para el abordaje y tratamiento efectivo en mujeres embarazadas, pueden ser aplicadas para rehabilitar - contener distintos síntomas durante el embarazo (Pérez-Miranda et al., 2021). 


\section{METODOLOGÍA}

El presente estudio es de corte descriptivo con un enfoque cuantitativo de diseño transversal, cuyo objetivo consistió en identificar acerca de los trastornos mentales en la etapa gestacional. En la recopilación de la información que permitió dilucidar el contexto de la investigación, formular los procesos de ejecución y describir el fenómeno en las partes que lo componen, se tomó en consideración toda la bibliografía necesaria relacionada a la investigación que abordan la temática en estudio. Se tomó en cuenta varias fuentes de información de diferentes autores, como libros, artículos, ensayos, publicaciones en páginas web, entre otros.

La muestra fue conformada por las mujeres embarazadas que fueron atendidas por control de rutina en el Hospital Básico Natalia Huerta de Niemes del cantón Rocafuerte desde el primer trimestre del año 2021, cuyas edades oscilan de los 18 a los 35 años.

Como criterios de inclusión se tomaron en consideración que las participantes se encuentren dentro del rango etario a estudiar, que deseen participar en el estudio y que tengan su ficha en el sistema del hospital. Como criterios de exclusión se dejaron fuera del estudio aquellas mujeres que no querían participar, mujeres menores de 18 años o mayores de 35 y personas con patologías psiquiátricas conocidas.

La evaluación se efectuó a través de la toma de información de las fichas de las pacientes y la aplicación del Cuestionario de Síntomas "SelfReporting Questionnaire" (SRQ) un instrumento propuesto por la Organización Mundial de la Salud para estudios con la población en la atención primaria a la salud.

En torno a los aspectos éticos, se ha realizado el uso del consentimiento informado, de la misma manera el autor declara no tener ningún conflicto de interés, y para el procesamiento de datos, se utilizó el software estadístico SPSS.

\section{DESARROLLO}

La salud mental se relaciona con las condiciones sociales, las mujeres se encuentran en estado alto de riesgo cuando tienen un trabajo sin remuneración, como tareas del hogar y cuidado de la familia; lo que conlleva muchas veces a que no 
logren tener un buen nivel socioeconómico que les permitan acceder a protección, actividades de emprendimiento, educación, un empleo digno y con remuneración justa, o incluso participar plenamente en política.

Esta investigación tiene como fin conocer e identificar los trastornos mentales presentes en la población participante. Esta evaluación se realizó mediante la información de las fichas de las pacientes ingresadas en el Hospital Básico Natalia Huerta de Niemes, mediante la aplicación del Cuestionario de Síntomas "Self-Reporting Questionnaire".

En este estudio se contó con la participación de 100 mujeres gestantes, con una edad media de 23,8 años, de las cuales un $82 \%$ se encontraba en unión libre en un $82 \%$. Debido a la zona rural donde viven estas mujeres, solo un $17 \%$ ha alcanzado la educación superior, siendo un $78 \%$ de las mismas bachilleres. Solamente un $21 \%$ de las participantes tenían un trabajo remunerado, el resto se quedan en casa.

Dentro de su periodo de gestación un $22 \%$ de las participantes estaba en su primer trimestre, un $38 \%$ en el segundo y un $40 \%$ en su tercer trimestre. De estas solamente un $18 \%$ habían planificado su embarazo, en un $10 \%$ de estos casos, eran multíparas con 4 a 5 embarazos.

Los resultados obtenidos como temprana edad, baja escolaridad, la no planificación familiar, pocas ocupaciones a nivel laboral nos prescriben factores de riesgos relacionados al padecimiento de alteraciones a nivel de salud mental, siendo estos factores predisponentes o desencadenantes en la depresión durante y posterior al parto. 
Gráfico № 1. Encuesta psicosocial.

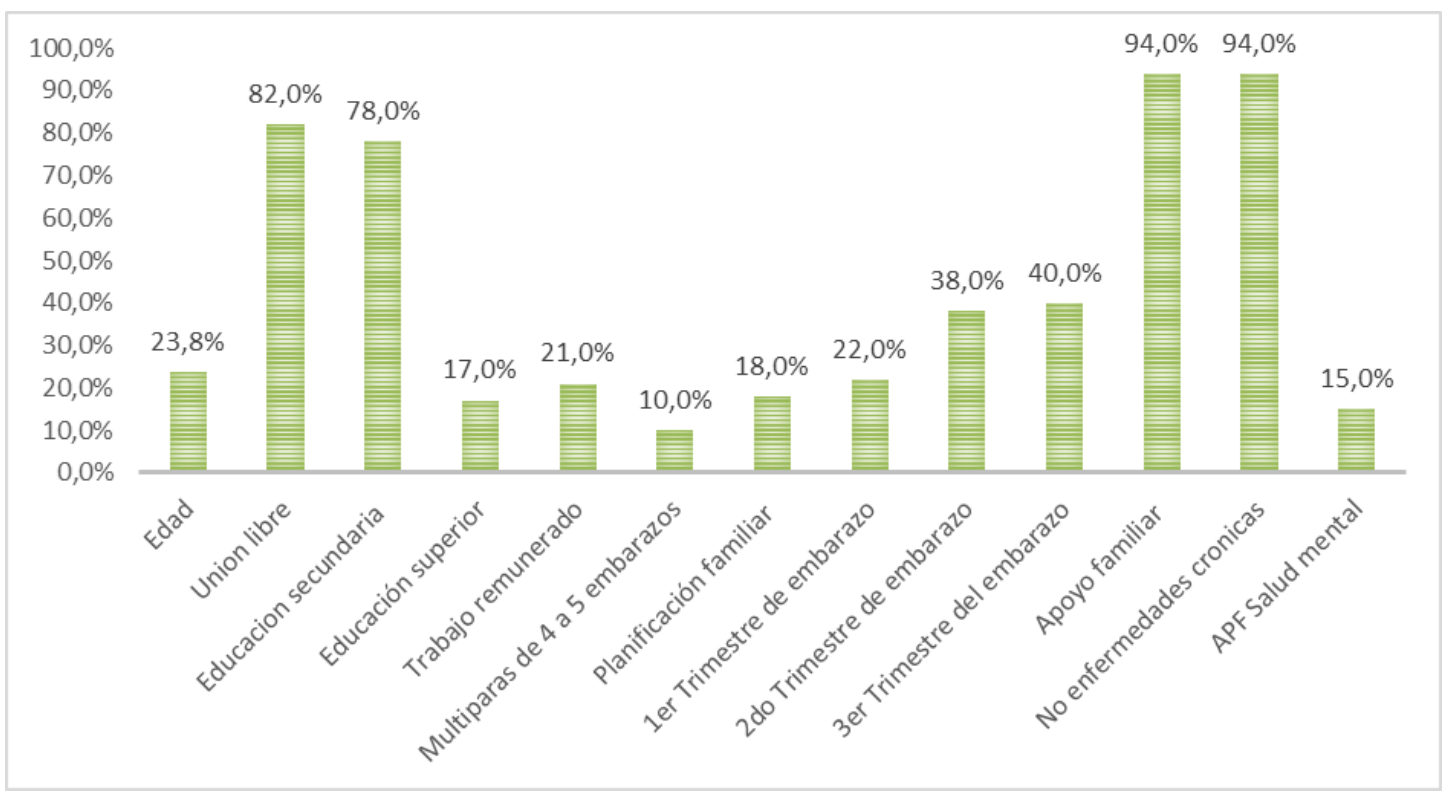

Fuente: Las participantes.

Gráfico № 2. Cuestionario de síntomas (SRQ).

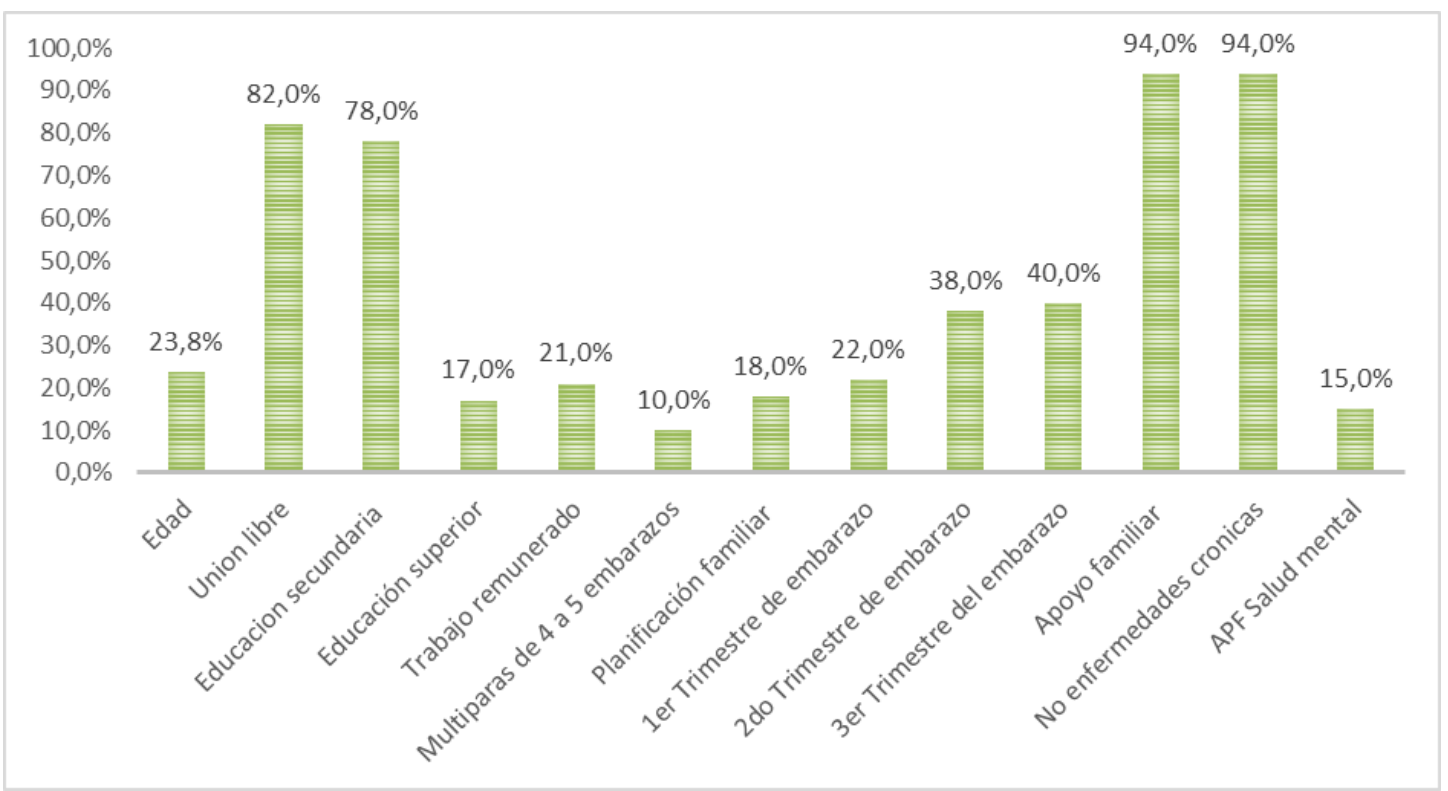

Fuente: Las participantes.

En el análisis del cuestionario de Síntomas "Self-Reporting Questionnaire" (SRQ), se obtiene como resultado más relevante, que el $78 \%$ de la población encuestada refiere tener síntomas de nerviosismo, tensión y aburrimiento, mientras que el $62 \%$ de la población reporto tener sensaciones desagradables a nivel gástrico, en compatibilidad con la pregunta número (7) del cuestionario de síntomas (SRQ), que también muestra relación en torno a 
alteraciones gástricas con un porcentaje de $58 \%$. Otros síntomas como el trastorno del ciclo vigilia sueño se ve reflejado en un porcentaje del $57 \%$ y cefaleas recurrentes en un $53 \%$ de las mujeres encuestadas.

En menor porcentaje se identificaron síntomas relacionadas con el estado de ánimo como la tristeza en un 33\% y la dificultad para disfrutar de las actividades de la vida diaria en un $35 \%$. El $35 \%$ de las mujeres embarazadas refirieron haber tenido ideas recurrentes de querer acabar con su vida, acompañados en un $29 \%$ de labilidad emocional y cansancio psicofísico en un $28 \%$. En una minoría de la muestra estudiada el $3 \%$ de mujeres embarazadas tienen antecedentes y síntomas relacionados con la epilepsia.

Toda esta sintomatología descrita guarda estrecha relación con los trastornos de ansiedad (CIE10F411) y depresión (CIE10-F320).

Como dato interesante, un 9\% de las mujeres embarazadas puntuaron positivo en los ítems que valoran psicosis, en lo cual se contrarresta con la observación clínica y se concluye que su porcentaje se debe a características histriónicas en el momento de la aplicación de la encuesta. Vale recordar que la psicosis no es una alteración muy prevalente durante el embarazo, teniendo una prevalencia de $0.1 \%$ al $0.2 \%$ y suele acentuarse en periodos postparto, con síntomas como alucinaciones generalmente auditivas, pero a veces contacto visual también suele verse alterado, comportamientos perturbados $y$ anormales en relación a la paciente.

Así también se debe analizar que un $78 \%$ de la población de estudio refirió que su embarazo es no deseado por lo que las mujeres y sus parejas enfrentan decisiones difíciles $y$ tienen consecuencias en la salud y la situación socioeconómica de la mujer, de su pareja y de su familia. Del total de mujeres encuestadas, 72 (72\%) de ellas parió por medio de parto natural, y el resto, es decir 28 (28\%) se les realizó cesárea.

A partir de esos trastornos mentales se desarrollan en las mujeres a causa de los problemas reproductivos como falta de decisión reproductiva, embarazo no deseado, infecciones o enfermedades de transmisión sexual (ETS), 
infertilidad, riesgos en el embarazo. Situaciones que reflejan que la salud mental está ligada a la morbilidad maternal.

A nivel de salud pública en Ecuador no existe un protocolo o guía de atención especializado para el tratamiento de trastornos mentales en mujeres embarazadas, lo que tendría como consecuencia un mal manejo de la sintomatología y posibles repercusiones a largo plazo en la mujer y su hijo (Ruiz-Acosta et al., 2017). La mayor parte de trastornos mentales presentan protocolos de intervención, en donde se indican los tratamientos considerados como primera elección, muchos de estos tratamientos son de contención psicofarmacológica, mismos que en su mayoría, se encuentran contraindicados para las mujeres en estado de gestación. En estos casos se evalúa el riesgo-beneficio de la administración farmacológica, cuando el riesgo es mayor se abstienen de prescribirlos, por lo que estas alteraciones quedan sin ser tratadas de manera integral (Vera Carrasco, 2015).

\section{DISCUSIÓN}

En un estudio publicado por la revista clínica de la escuela de medicina de Costa Rica UCR-HSJD (2019) concluye que los trastornos mentales afectan a las personas desde temprana edad incluyendo a mujeres en edad reproductiva. Los pacientes que tienen otras patologías priorizan su enfermedad de base antes que la salud mental, lo que implica dejar al último la terapia psicológica y muchas de las veces, el tiempo ya no es suficiente para acudir a ellas (Calvo \& Jaramillo, 2015).

El embarazo es uno de los cambios biológicos que presenta mayor prevalencia para la enfermedad mental, sin embargo, se discute que en realidad existan figuras psiquiátricas producidas por este cambio biológico. Lo que si está determinado es que las mujeres gestantes, por el mismo hecho de los cambios que experimentan, presentan un alto grado de vulnerabilidad, por lo que un mal diagnóstico 0 un tratamiento inadecuado conlleva serios riesgos para la vida de la madre y de su hijo.

El Manual diagnóstico y estadístico de los trastornos mentales (DSM-IV- 
TR) no contempla categorías diagnósticas específicas para la depresión o para la psicosis observada durante el embarazo 0 durante el año posterior al parto. Para este sistema nosológico tanto la depresión como la psicosis asociada al embarazo y al parto serían crisis depresivas o psicóticas ligadas a otro tipo de enfermedad mental, por ejemplo, al trastorno afectivo bipolar (Castaño-Henao, 2005).

Se establece que el puerperio es uno de los periodos de mayor riesgo en la presentación de trastornos del estado de ánimo. Cerca del $85 \%$ de las mujeres presentan algún tipo de alteración del estado de ánimo durante el posparto, Algunas mujeres experimentan síntomas transitorios y leves, pero un porcentaje considerable, hasta el $25 \%$ de las mujeres manifiestan algún tipo de trastorno incapacitante y persistente. En este último grupo, hasta un $50 \%$ de los casos permanecen sin diagnosticar (Regier et al., 2000).

Los trastornos mentales que tienen relación con la etapa reproductiva de la mujer están prescritos por múltiples factores, desarrollándose en las diferentes fases del embarazo, convirtiendo aquello como un verdadero factor de riesgo entorno a la salud mental de la madre y del niño/a. Nuestra población, al igual que otras poblaciones que ya han venido experimentando esta problemática, no está exenta de esta patología, pero se diferencian en que éstas oportunamente han logrado realizar propuestas de cambios en el campo de la salud mejorando la visión y abordaje a esta patología. Cabe indicar que, como investigador, afirmo que la identificación es el primer paso clave al tratamiento, pero hay que enfocarse en mejorar la cosmovisión de la problemática.

Un estudio realizado por el Instituto Nacional de Perinatología Isidro Espinoza de los Reyes de México (2011), concluye con una propuesta para el campo de la salud, acerca de trastornos mentales en mujeres en edad reproductiva, refiriendo su etiología principal a fases lúteas del ciclo menstrual y vulnerabilidad fisiopatológica, orientando esta propuesta a la prevención mediante tratamientos hormonales efectivos enfocados al abordaje psiquiátrico y 
a la adaptación hormonal para contribuir a la no aparición de diversos trastornos. Refiero que, para el tratamiento de los trastornos mentales es fundamental trabajar en la prevención, pero desde una perspectiva multidisciplinaria que involucre ciencias accesibles a los principales beneficiarios que son las pacientes que padecen este tipo de alteraciones.

La depresión en las mujeres es más común que en los hombres, pero la causa específica se desconoce en muchos casos y no se encuentra un factor causal directo. Parece ser el resultado de una combinación de factores como predisposición genética, historia familiar, cambios bioquímicos que responden a un desequilibrio de neurotransmisores, factores del ambiente y estrés, factores hormonales y reproductivos, factores psicológicos y sociales entre otros factores que pueden ser determinantes (Regier et al., Op. cit., 2000).

En la mujer específicamente se presenta el síndrome disfórico premenstrual, depresión en el embarazo y depresión posparto. El síndrome disfórico premenstrual implica síntomas afectivos como labilidad; conductuales, como cambios en los patrones de alimentación; y físicos como edema, cefalea e hipersensibilidad mamaria. Este patrón aparece en un momento específico de cada ciclo menstrual y desaparece durante cierto tiempo entre ellos. Existe también la depresión en el embarazo, la cual se presenta en forma severa en una de cada diez mujeres embarazadas. Durante el puerperio, factores bioquímicos y estrés psicológico pueden desencadenar trastornos psiquiátricos en la mujer y ocasionar la depresión posparto (Salinas, 1994).

Diversos estudios plantean que la enfermedad mental durante el embarazo y la lactancia se relaciona con los cambios que las hormonas producen sobre el afecto, el pensamiento y el comportamiento de la mujer, los cuales se manifiestan como alteraciones depresivas, psicosis posparto, reactivación de trastornos mentales psicóticos, estados disociativos 0 confusionales. Tales estudios tienden a considerar que existen entidades psiquiátricas específicas desencadenadas por los cambios hormonales producidos durante el 
embarazo y el parto, las cuales se asentarían en personalidades vulnerables. Se ha observado, por ejemplo, que la depresión posparto se presenta en $10 \%-20 \%$ de las mujeres estadounidenses en los primeros seis meses y que cerca de la mitad de estas mujeres aún presentan síntomas depresivos un año después (Castaño-Henao., Op. cit., 2005).

En otro sentido, un estudio publicado en la revista argentina de psiquiatría Vertex (2018), se determina que la deficiencia del entorno social, familiar y educativo contribuye en la aparición de alteraciones mentales. Además, se puede determinar que los conflictos psicosociales son en sí mismos factores de riesgo para empeoramientos de la salud mental $y$ se convierten en las principales necesidades a abordar en los grupos vulnerables.

Además, es necesario considerar a los trastornos por abuso de sustancias, éstos están íntimamente asociados con los trastornos de personalidad y eso lleva a que el paciente incumpla con las sesiones. Este tipo de pacientes son más complicados de tratar, porque influye mucho el soporte social y laboral; la necesidad de consumo o adicción; problemas económicos y la comorbilidad con otras enfermedades, que en conjunto hacen que no acudan a la terapia psicológica, y, por ende, no reciben un tratamiento adecuado (Jotak \& Almao, 2007).

Entonces, se debe tener en cuenta que la maternidad es una función que lleva tiempo para establecerse, en este periodo se despliegan conductas psicológicas que conllevan a una adaptación física y la creación de lazos afectivos con el recién nacido. Las posibles patologías acontecidas en este periodo son un motivo frecuente de consulta en urgencias, siendo incluso una de las causas más frecuentes de mortalidad materna (Sesmero, 2002).

Estudios muestran dos consecuencias importantes que atentan contra la integridad del individuo. La primera, la más importante y la más peligrosa, es el suicidio. Los pacientes que sufren depresión tienen cuatro veces más riesgo de suicidio que la población general. El $6 \%$ de las personas 
diagnosticadas de depresión cometerán suicidio (Calvo \& Jaramillo, 2015). El riesgo de suicidio aumenta hasta 20 veces en los pacientes con las formas más severas de la enfermedad. Los pacientes con un episodio de depresión actual, entre un $20 \%$ y un $25 \%$ intentarán suicidarse. Si la depresión no se trata incrementa el riesgo de suicidio; por el contraste con un adecuado tratamiento, se reduce a la mitad el riesgo del suicidio (Bousoño et al., 2008).

Se estima que alrededor que del 10 al $15 \%$ de las madres sufre de depresión posparto y que aproximadamente 2 de 1000 mujeres presenta psicosis durante el primer año de vida de su hijo. La presencia de síntomas mentales graves durante el embarazo o en el posparto son de gran importancia, no sólo por su asociación con los indicadores de morbi-mortalidad, sino también, por las consecuencias y sus posibles complicaciones (A. Rossler \& Hofecker, 2003).

Así también se debe analizar que un $78 \%$ de la población de estudio refirió que su embarazo es no deseado por lo que las mujeres y sus parejas enfrentan decisiones difíciles y tienen consecuencias sobre la salud y la situación socio-económica de la mujer, su pareja y su familia. De ahí que en los últimos años se viene intentando optimizar el diagnóstico y poner a disposición tratamientos tempranos y eficientes para prevenir y tratar estos trastornos.

La quinta edición de la Guía de consulta de los Criterios Diagnósticos del DSM-5 menciona que los episodios del estado de ánimo pueden iniciar durante el embarazo o en el posparto, sin embargo, se diferencian según el período. Después del parto, entre un $3 \%$ y un $6 \%$ de las mujeres experimentarán el inicio de un episodio de depresión mayor durante el embarazo o en las semanas o meses que siguen al parto (American Psychiatric Association, 2014). Caracterizando que los trastornos afectivos posparto son aquellos trastornos que se inician durante las primeras cuatro semanas posteriores al parto. Dentro de estas se encuentran:

- "Baby blues" o síndrome del tercer día.

- Depresión posparto sin psicosis.

- Depresión posparto con psicosis. 
En mujeres en fase de puerperio, estas consideraciones se suman al hecho de que muchas mujeres en nuestro medio, por economía o cultura deciden dar de lactar a su recién nacido, por lo que la elección de un tratamiento farmacológico para ellas se debe indicar cuando está en peligro la vida de la madre, del niño o los síntomas son tan intensos que se justifique.

En relación al tratamiento farmacológico, existen multitud de psicofármacos antidepresivos que han demostrado ser eficaces para el tratamiento de la depresión. Su uso está especialmente indicado cuando los síntomas físicos son intensos y los pensamientos suicidas continuos. Si el ciclo del sueño y el apetito están muy alterados, el paciente muestra inquietud interna e insoportable $\mathrm{o}$, al contrario, presenta fatiga que le cuesta pensar $y$ moverse, sería recomendable que acudiese a una valoración médica especializada con el fin de evaluar la necesidad de tomar una medicación antidepresiva que estabilizara estos síntomas. La medicación va a remitir la sintomatología, pero debe ser integral el tratamiento para poder elaborar la toma de decisiones, alcanzar estabilidad emocional o generar habilidades que permitan afrontar la situación global.

De toda esta investigación, se verifica que existen protocolos de intervención a nivel general acerca de la depresión, como la Guía de Práctica Clínica del Diagnóstico y Tratamiento del Episodio Depresivo y del Trastorno Depresivo Recurrente en Adultos del Ministerio de Salud Pública del Ecuador (2017). Sin embargo, a nivel especifico (depresión durante y posterior al embarazo) no se cuenta con un protocolo de detección el cual incluya estrategias de intervención y abordaje de ésta. Se deben realizar nuevas investigaciones que puedan determinar si los trastornos mentales son más prevalentes cuando los embarazos son no deseados, lo que podría interpretarse como un problema psicosocial más que uno relacionado directamente con los cambios que suceden en el embarazo.

\section{CONCLUSIONES}

Se concluye que la mujer en edad reproductiva con un embarazo no deseado y otros factores de riesgo 
contribuyen a la aparición de alteraciones en su estado de salud mental, siendo la ansiedad y depresión las principales morbilidades para esta población vulnerable.

Se estima que la aplicación de herramientas psicométricas más específicas en torno a la búsqueda de alteraciones mentales en mujeres en edad gestacional contribuiría a una identificación y tratamiento oportuno de estos trastornos, mejorando su calidad de vida durante su embarazo, posparto y en etapas posteriores.

Se identifica la importancia de una valoración multidisciplinaria entre el médico y el psicólogo ya que esta puede contribuir a mejorar los factores de riesgo asociados a ciertos trastornos mentales, la planificación familiar, el número de hijos y otros factores psicosociales.

Es importante realizar una intervención integral de la depresión, que no solo esté orientado en etapas posnatales, si no, más bien este dirigido a la detección temprana y tratamiento de ésta, desde el sector salud, partiendo de estos ensayos clínicos que acortando la brecha entre la problemática y su tratamiento.

El presente estudio pone en evidencia la necesidad de un protocolo multidisciplinario para la atención de mujeres en etapa de gestación, con especial atención a aquellas que presentan factores de riesgo para padecer un trastorno mental o para aquellas que ya lo padezcan.

\section{BIBLIOGRAFÍA}

A. Rossler, \& Hofecker, M. (2003). Postpartum depression: Do well still need the diagnostic term. 51-56.

American Psychiatric Association (Ed.). (2014). Guía de consulta de los criterios diagnósticos del DSM-5. American Psychiatric Publishing.

Bousoño, M., Baca, E., Álvarez, E., Eguiluz, I., Martín, M., \& Roca, M. (2008). Complicaciones de la depresión a largo plazo. Actas Esp Psiquiatr, 36, 4452.

Calvo, J., \& Jaramillo, L. (2015). Detección del trastorno depresivo mayor en atención primaria. Revista de la Facultad de Medicina, 63(3), 472-482.

https://doi.org/10.15446/revfa cmed.v63n3.46424 
Castaño-Henao, B. L. (2005). TRASTORNO MENTAL Y FILICIDIO. Revista Colombiana de Psiquiatría, 34, 82-89.

González, A. (2015). Salud Mental de la mujer en edad gestacional. 36 .

Guimaraes, F. J., Santos, F. J. D. S., Leite, A. F. B., Holanda, V. R. D., Sousa, G. S. D., \& Perrelli, J. G. A. (2019). Adoecimiento mental en gestantes. Enfermería Global, 18(1), 499-534.

https://doi.org/10.6018/egloba I.18.1.328331

Instituto Nacional de Perinatología Isidro Espinoza de los Reyes de México. (2011). Los trastornos mentales relacionados a la edad reproductiva de la mujer: Una nueva propuesta en el campo de la salud mental. Gaceta Médica de México., 5.

Jotak \& Almao. (2007). Abandono del tratamiento en una consulta externa de Psiquiatría y Psicología Clínica. Revista electrónica de PortalesMedicos.com. http://www.portalesmedicos.c om/monografias/abandono_tr atamiento/index.htm

Ministerio de Salud Pública del Ecuador. (2017). Guía de Práctica Clínica del Diagnóstico y Tratamiento del Episodio Depresivo y del
Trastorno

Depresivo Recurrente en Adultos.

Nariño, D. (2017). Epilepsia y embarazo.

Organización Mundial de la Salud. (2019). Trastornos mentales. Trastornos mentales. https://www.who.int/es/newsroom/factsheets/detail/mentaldisorders

Organización Panamericana de la Salud. (2017, marzo 30). «Depresión: Hablemos», dice la OMS, mientras la depresión encabeza la lista de causas de enfermedad. Pan American Health Organization / World Health Organization. https://www3.paho.org/hq/ind ex.php?option=com_content\& view=article \&id $=13102$ :depre ssion-lets-talk-says-who-asdepression-tops-list-ofcauses-of-illhealth\&ltemid=1926\&lang=es

Organización Panamericana de la Salud/OMS. (2020). "No hay salud sin salud mental"OPS/OMS | Organización Panamericana de la Salud. https://www.paho.org/es/notic ias/8-10-2020-no-hay-saludsin-salud-mental

Pérez-Miranda, G., Ortiz-Pinilla, D., Niño-Mancera, D. A., GómezSalcedo, L. H., \& BayonaGranados, K. V. (2021). Eficacia de las intervenciones no farmacológicas en la prevención de la depresión 
posparto: Una revisión sistemática de los resultados en gestantes con y sin factores de riesgo. Médicas UIS, 34(1), 73-90.

Pérez-Solís, D., Rodríguez-Vargas, M., Rodríguez-Ricardo, A., Pérez-Solís, D., RodríguezVargas, M., \& RodríguezRicardo, A. (2020). Prevalencia de trastorno mental en embarazadas. Revista Archivo Médico de Camagüey, 24(4). http://scielo.sld.cu/scielo.php? script=sci_abstract\&pid=S102 5 02552020000400004\&lng=es \&nrm=iso\&tlng=pt

Regier, D. A., Farmer, R. D., Lock, B., Keith, S., Judo, L., \& Godwin, F. (2000). Comorbility of mental disorders with alcohol an other drug abuse: Results from Epidemiologic catchment area (ECA) study. JAMA, 264.

revista clínica de la escuela de medicina de Costa Rica UCRHSJD. (2019). Trastorno bipolar en el embarazo. 8 .

Ruiz-Acosta, G. J., OrganistaGabriel, H., López-Avendaño, R. A., Cortaza-Ramírez, L., \& Vinalay-Carrillo, I. (2017). Prevalencia de Depresión en Embarazadas de una Institución de Salud de Primer Nivel. Revista Salud y Administración, 4(12), 25-30.
Sainz Aceves, K. G., Chávez Ureña, B. E., Díaz Contreras, M. E., Sandoval Magaña, M. A., \& Robles Romero, M. A. (2013). Ansiedad y depresión en pacientes embarazadas. Atención Familiar, 20(1), 2527.

https://doi.org/10.1016/S1405 $-8871(16) 30082-7$

Salinas, J. (1994). Relación entre el funcionamiento familiar, el estrés y la ansiedad. Revista de la Facultad de Medicina de los Andes, 175-179.

Sesmero, J. (2002). Mortalidad materna en España. En Programa Obstet. Ginecol (pp. 524-534).

Sutter-Dallay, A.-L., Scotta, G., Lemoigne, S., \& Gressier, F. (2020). Psiquiatría y embarazo: La madre y el hijo. EMC - GinecologíaObstetricia, 56(4), 1-13. https://doi.org/10.1016/S1283 $-081 \times(20) 44317-6$

Vera Carrasco, O. (2015). USO DE FÁRMACOS EN EL EMBARAZO. Revista Médica La Paz, 21(2), 60-76.

Vertex. (2018). EPIDEMIOLOGÍA EN PSIQUIATRÍA Y SALUD MENTAL. revista argentina de psiquiatría. 